Ann. Biol. anim. Bioch. Biophys., I969, 9 (1), гог-Iro.

\title{
INFLUENCE DE LA TEMPÉRATURE ET DU PH SUR QUELQUES CARACTÉRISTIQUES PHYSICO-CHIMIQUES DES PROTÉINES SARCOPLASMIQUES DU MUSCLE DE PORC CONSÉQUENCES TECHNOLOGIQUES.
}

\author{
J. CHARPENTIER \\ avec la collaboration technique de Denise Guene et Anne-Marie Guevgneau \\ Laboratoire de Recherches sur la Viande (1), \\ Centre national de Recherches zootechniques, 78 - Jouy-en-Josas \\ Institut national de la Recherche agronomique
}

\section{SOMMAIRE}

Cette expérience avait pour but de préciser l'influence respective de la température et du $\mathrm{pH}$ sur la solubilité des protéines sarcoplasmiques et sur les caractéristiques spectrales et électrophorétiques de la myoglobine du muscle Longissimus dorsi de porc.

Les protéines sarcoplasmiques sont incubées pendant 4 heures à des températures variables dans des solutions de tampon phosphate-citrate $0,2 \mathrm{M}$ ou phosphate $0,2 \mathrm{M}$ de façon à obtenir une gamme de $\mathrm{pH}$ comprise entre 5,5 et 6,7. Les protéines en solution sont déterminées par la méthode de Folin-Lowry. L'électrophorèsé en gel d'amidon est effectuée selon la technique de PouLIK. La dénaturation des protéines sarcoplasmiques est particulièrement importante lorsque le $\mathrm{pH}$ est compris entre 5,5 et 5,8 et lorsque la température est supérieure à $40^{\circ} \mathrm{C}$. L'électrophorèse sur gel d'amidon montre que les protéines dénaturées sont principalement la créatine kinase et les protéines à migration cathodique rapide.

L'étude des spectres d'absorption de la myoglobine montre que la conjonction d'un pH bas et d'une température élevée entraîne une oxydation et non une dénaturation de la myoglobine. D'un point de vue pratique, les résultats de cette expérience indiquent que lorsque la diminution du $\mathrm{pH}$ est particulièrement rapide, la réfrigération rapide des carcasses ne devrait pas permettre de pallier l'insuffisance de rétention d'eau et de coloration qui résulte précisément de la dénaturation des protéines musculaires. En revanche, dans le cas des muscles présentant une chute de $\mathrm{pH}$ relativement lente, la rapidité et l'intensité de la réfrigération peuvent alors exercer un effet bénéfique en abaissant suffisamment la température musculaire.

\section{INTRODUCTION}

A l'issue des nombreux travaux effectués ces dernières années, il apparaît que la faible rétention đ'eau et à un moinđre degré, l'insuffisance de coloration des viandes exsudatives, sont dues à une glycogénolyse post-mortem particulièrement rapide.

(1) Nouvelle adresse : Station de Recherches sur la Viande, C. R. Z. V., Theix, 63-Clermont-Ferrand. 
Le $\mathrm{pH}$ musculaire atteint en effet sa valeur ultime très peu de temps après l'abattage, alors que la température du muscle est voisine de celle de l'animal vivant. Cette conjonction d'un $\mathrm{pH}$ bas et d'une température relativement élevée entraîne la précipitation d'une partie des protéines sarcoplasmiques. Selon BENDALI et WiSMERPederRsen (I962), les protéines sarcoplasmiques dénaturées précipiteraient sur les protéines myofibrillaires, ce qui aurait pour effet de réduire également la solubilité de ces dernières et de masquer des groupements hydrophiles, donc par là même, de diminuer la rétention d'ea11. Par ailleurs, les protéines sarcoplasmiques dénaturées masqueraient en quelque sorte la myoglobine et réduiraient ainsi son pouvoir colorant (MC LOUGHLIN et GoLDSPINK, I963). L'interaction du $\mathrm{pH}$ et de la température se traduit donc par des modifications des propriétés physico-chimiques des protéines musculaires qui sont responsables en définitive de caractéristiques technologiques importantes, telles que la rétention d'eau et la coloration. Aussi nous a-t-il semblé souhaitable de préciser les modalités d'action de ces deux paramètres sur, d'une part, la solubilité des protéines sarcoplasmiques et, d'autre part, l'état physico-chimique de la myoglobine.

\section{MATÉRIEL E'T MÉ'THODES}

Un échantillon d'un poids de 50 grammes environ était prélevé, sitôt la saignée terminée, sur le muscle Longissimus dorsi de porcs Large White pesant entre roo et $110 \mathrm{~kg}$. Un homogénat était réalisé par broyage du tissu musculaire dans 3 volumes d'eau distillée à I 6000 tours/minute pendant une minute à l'aide d'un mixeur Biorex. L'homogénéisat était maintenu pendant 6 heures à $0^{\circ} \mathrm{C}$, puis centrifugé à $600 \mathrm{~g}$ pendant 20 minutes. Des parties aliquotes de surnageant étaient diluées avec 3 volumes de tampon phosphate-citrate $0,2 \mathrm{M}$ ou phosphate $0,2 \mathrm{M}$, de façon à obtenir une gamme de pH comprise entre 5,5 et 6,7. La teneur en azote de ces solutions était déterminée par la méthode de LowRy et al. (I95I). Le spectre d'absorption était déterminé entre 440 et $660 \mathrm{~m} \mu$ à l'aide d'un spectrophotomètre SAFAS. L'analyse électrophorétique des protéines en solution était effectuée au moyen de l'électrophorèse en gel d'amidon selon la technique discontinue de Poulik (I957). Un tampon $0,076 \mathrm{M}$ tris- $0,005 \mathrm{M}$ acide citrique $\mathrm{pH} 8,65$ était utilisé pour la préparation du gel. Le tampon circulant consistait en un tampon borate de sodium $0,3 \mathrm{M} \mathrm{pH} \mathrm{8,4.} \mathrm{La} \mathrm{durée} \mathrm{de} \mathrm{l'electrophorèse}$ était de 5 heures 30 avec un champ électrique de 6 volts $/ \mathrm{cm}$. Les protéines étaient colorées par l'amidoschwartz. La myoglobine était mise en évidence par sa réaction peroxydasique donnant une coloration brune avec la benzidine (MORETTI et al., I957). Les électrophorégrammes étaient photographiés dès leur sortie des bains de coloration.

Des fractions de $5 \mathrm{ml}$ des solutions étaient mises à incuber à des températures variables comprises entre 20 et $45^{\circ}$ pendant des durées variant de une à six heures. Une durée de 4 heures était choisie pour les déterminations n'impliquant pas une étude cinétique de la solubilité en fonction du temps.

Sitôt l'incubation terminée, les solutions étaient centrifugées à $600 \mathrm{~g}$ pendant 20 minutes. Les mêmes déterminations que précédemment étaient effectuées sur les surnageants. A partir des dosages de protéine avant et après incubation, on calculait le pourcentage de protéines précipitées.

L'étude concernant l'influence du pH et de la température sur les caractéristiques spectrales et "lectrophorétiques de la myoglobine était réalisée sur une fraction isolée par chromatographie sur carboxymethyl-cellulose (AWAD et KoTITE, 1966). Après broyage de ro grammes environ de tissu musculaire dans 3 volumes de tampon phosphate $0,0 \mathrm{I} \mathrm{M} \mathrm{pH} \mathrm{6,2} \mathrm{et} \mathrm{centrifugation} \mathrm{à} 600 \mathrm{~g}$ pendant 20 minutes, l'extrait ainsi obtenu était chromatographié sur une colonne de carboxynéthylcellulose équilibrée avec le même tampon phosphate $0,0 \mathrm{I} \mathrm{M} \mathrm{pH} 7,3$. La bande contenant la myoglobine était éluée avec un tampon phosphate o,1 M pH 7,3. L'addition d'acide acétique $0,2 \mathrm{M}$ permet tait d'obtenir une gamme de $\mathrm{pH}$ comprise entre 7,3 et 5,5. Les solutions ainsi obtenues étaient mises à incuber à des températures variables comprises entre 20 et $45^{\circ}$ pendant des durées variant de $\mathrm{I}$ à 4 heures.

A l'issue de l'incubation, le spectre d'absorption était déterminé entre 440 et $660 \mathrm{~m} \mu$ à l'aide d'un spectrophotomètre SAFAS. L'analyse électrophorétique de la myoglobine était conduite selon la technique MORETTI et al. (1957). 


\section{RÉSULTATS ETT DISCUSSION}

I. Infuence de la température et du $p H$ sur la solubilité des protéines sarcoplasmiques.

I. I. Solubilité des protéines sarcoplasmiques en fonction de la durée d'incubation.

La figure I montre nettement que l'évolution de la solubilité des protéines sarcoplasmiques en fonction de la durée d'incubation dépend étroitement du $\mathrm{pH}$ et de la température. Ainsi, la précipitation est particulièrement rapide pour un $\mathrm{pH}$ de 5,5

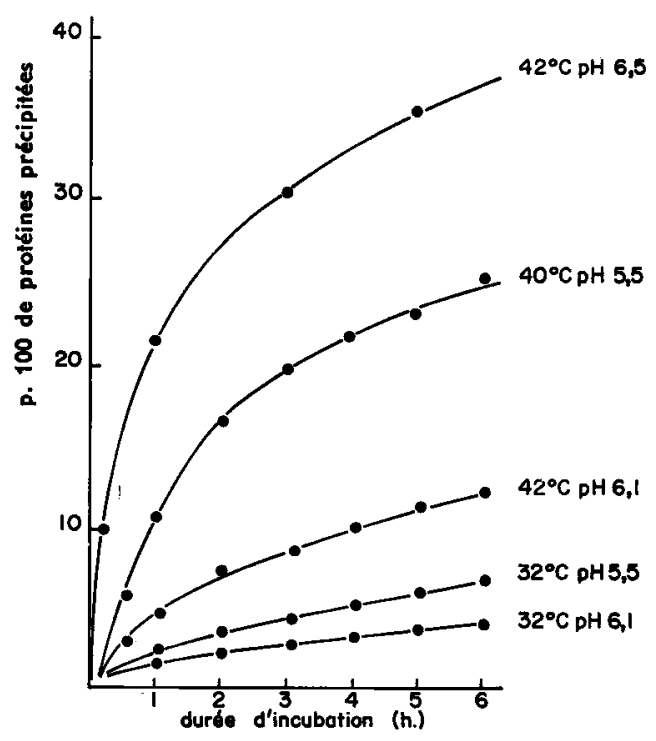

FIG. I. - Précipilation des protêines sarcoplasmiques a différents $p H$ et différentes températures, en fonction de la durée d'incubation

et une température de $42^{\circ} \mathrm{C}$. Cette condition est précisément celle que l'on peut rencontrer dans le muscle de porc exsidatif dans l'heure qui suit l'abattage. Il convient de remarquer que, pour une température de $42^{\circ} \mathrm{C}$ et un $\mathrm{pH}$ de $6, \mathrm{I}$, la précipitation est progressive, mais néanmoins limitée. De même, lorsque la température est de $32^{\circ} \mathrm{C}$ par exemple, la précipitation est très lente et peu importante quelle que soit la valeur $\mathrm{du} \mathrm{pH}$. Compte tenu de la cinétique de la solubilité en fonction du temps, nous considérons dans la suite de cette étude une durée d'incubation de 4 heures. Cette durée correspond, par ailleurs, sur le plan pratique, au délai pendant lequel la température de la majeure partie de la carcasse est supérieure à $30^{\circ} \mathrm{C}$ dans des conditions de réfrigération variées. 
I. 2. Solubilité des protéines sarcoplasmiques dans le cas d'une durée d'incubation de 4 heures.

La figure 2 montre l'évolution du pourcentage de protéines précipitées en fonction de la température d'incubation et à différents $\mathrm{pH}$. La dénaturation des protéines sarcoplasmiques est particulièrement importante lorsque le $\mathrm{pH}$ est compris entre 5,5 et 5,8 et lorsque la température est supérieture à $40^{\circ} \mathrm{C}$. La confrontation de nos résultats et de ceux obtenus par Scopes (I964) dans des conditions expérimentales semblables, mais avec des solutions de protéines sarcoplasmiques de bœuf, montre que, pour des $\mathrm{pH}$ inférieurs à 6,00 , les protéines du porc sont beaucoup plus sensibles à l'action de la température, ce qui contribue vraisemblablement à expliquer en partie que les viandes exsudatives se rencontrent principalement dans l'espèce porcine.

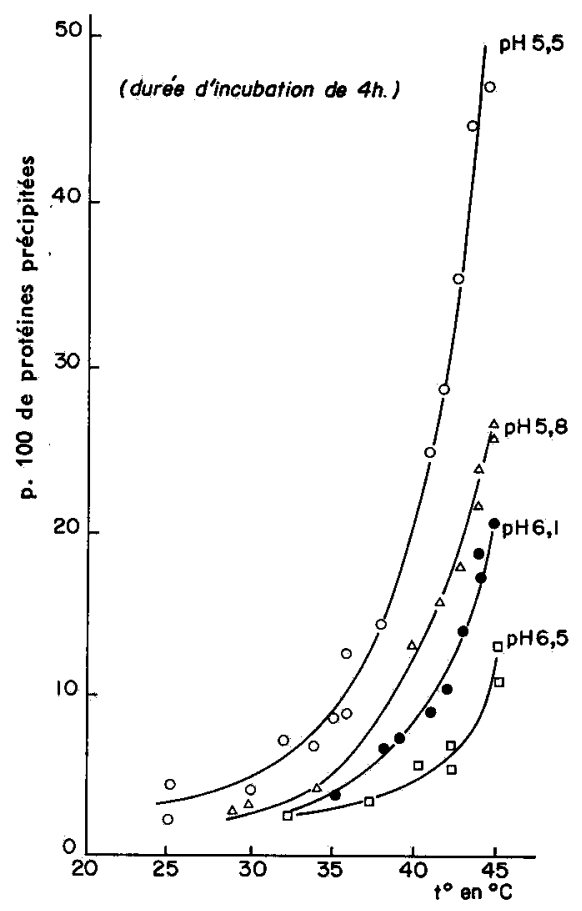

FIG. 2. - Précipitation des protéines sarcoplasmiques à différents $p H$ et da différentes températures après 4 heures d'incubation

\section{3. Nature de la fraction précipitée.}

L'étude électrophorétique des protéines sarcoplasmiques montre que la conjonction d'un bas $\mathrm{pH}$ et d'une température élevée entraîne systématiquement l'atténuation, voire la disparition des bandes cathodiques les plus rapides, ainsi que la diminution d'intensité de la bande anodique correspondant à la créatine kinase. La zone difficilement différenciable située entre les bandes, correspondant à la créatine kinase et à la myoglobine est également, en général, plus discrète. 
L'analyse détaillée de l'influence respective du $\mathrm{pH}$ et de la température (fig. 3) montre que les bandes cathodiques sont relativement plus sensibles à l'action de la température, alors que la bande correspondant à la créatine kinase semble plus

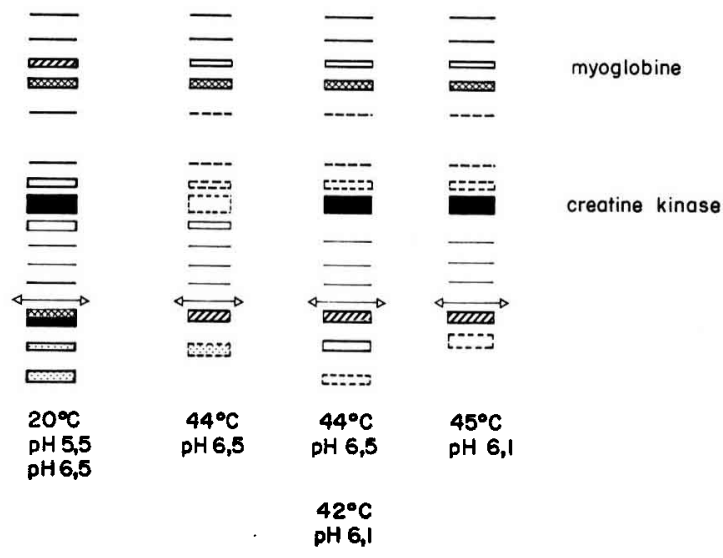

Fig. 3. - Élecirophorégramme des surnageants de solutions de protéines sarcoplasmiques obtenus aprés incubation dans différentes conditions de température et de $p H$ (coloration par l'amidoschwartz)

affectée par la diminution de $\mathrm{pH}$ que par l'augmentation de la température. Nos résultats confirment donc également chez le Porc l'action dénaturante de la température et $\mathrm{du} \mathrm{pH}$ vis-à-vis de la créatine kinase, déjà mise en évidence par ScOPES (I964). La dénaturation de la créatine kinase serait susceptible d'expliquer, en partie du moins, la rapidité de l'installation de la rigidité cadavérique dans le muscle exsudatif. En effet, la synthèse d'ATP à partir de la phosphocréatine et de l'ADP se trouverait inhibée et, par suite de l'activité ATPasique intense qui caractérise les muscles exsudatifs (BENDALL et al., I963), la déplétion de 1'ATP serait ainsi accélérée, La nature des bandes cathodiques n'est que très partiellement connue. Selon ScOPE, (I964), la fraction "Globulin X " représenterait une part importante des protéines qui migrent vers la cathode. Certes, dans nos conditions expérimentales (extraction aqueuse), nous ne devons extraire que partiellement la fraction "Globulin X ", compte tenu de la force ionique de l'homogénat.

Néanmoins, il semble logique de supposer que l'atténuation ou même la disparition de bandes cathodiques implique vraisemblablement une dénaturation des globulines. Cette hypothèse, déjà émise par KRZYwICKI et WISMER-PEDERSEN (I962), est étayée par de nombreux arguments. D'une part, le point isoélectrique de la fraction "Globulin X " est voisin de 5,5. L'abaissement du pH doit donc provoquer la coagulation de ces protéines. Leur point isoélectrique étant pratiquement analogue à celui des protéines myofibrillaires, il est donc logique de supposer qu'elles se condensent en quelque sorte avec ces dernières et réalisent ainsi une inhibition stérique de l'aptitude à la rétention d'eau. D'autre part, les différences d'extratibilité de pro-

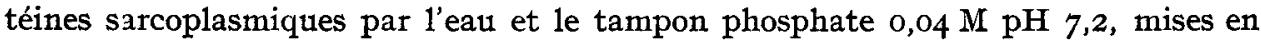
évidence par GouteFongeA ( 1967 ), dans le cas de muscles normaux et de muscles exsudatifs laisse supposer que la dénaturation consécutive à l'interaction pH-température porte effectivement sur la fraction " Globulin X ". Par ailleurs, compte tenu 
de la particulière sensibilité à la diminution de $\mathrm{pH}$ de la fraction désignée couramment par l'appellation " fraction $\mathrm{pH}_{5}$ ", il semble logique que les divers enzymes inclus dans cette fraction soient inévitablement affectés. Leur dénaturation expliquerait vraisemblablement la diminution d'intensité des électrophorégrammes constatée dans la zone située entre la créatine kinase et la myoglobine. Lors d'une étude antérieure sur le comportement électrophorétique des protéines sarcoplasmiques de muscles de porcs normaux et exsudatifs (ChARPENTIER et GouTEFongea, I963), nous n'avions pu mettre en évidence des différences dans les électrophorégrammes des protéines de ces deux types de muscle. Ceci ne saurait nous surprendre, car pour être décelables sans ambiguïté, les diminutions d'intensité des bandes d'électrophorèse ne peuvent être obtenues que dans des conditions de température et de $\mathrm{pH}$ suffisamment drastiques.

\section{Infuence de la température et du $p H$ sur l'état physico-chimique de la myoglobine.}

\section{I. Spectre d'absorption.}

Un floculat incolore plus ou moins abondant se forme lors de l'incubation des extraits musculaires. Son importance et la rapidité avec laquelle il se développe dépendent de la température et du $\mathrm{pH}$. En même temps que le floculat s'intensifie, la coloration s'atténue et la teinte se modifie. Les spectres d'absorption des surnageants d'extraits centrifugés après incubation à température élevée et à bas $\mathrm{pH}$ se caractérisent par une diminution des densités optiques consécutive à l'élimination d'une partie des protéines sarcoplasmiques et par l'apparition de maxima à 500 et

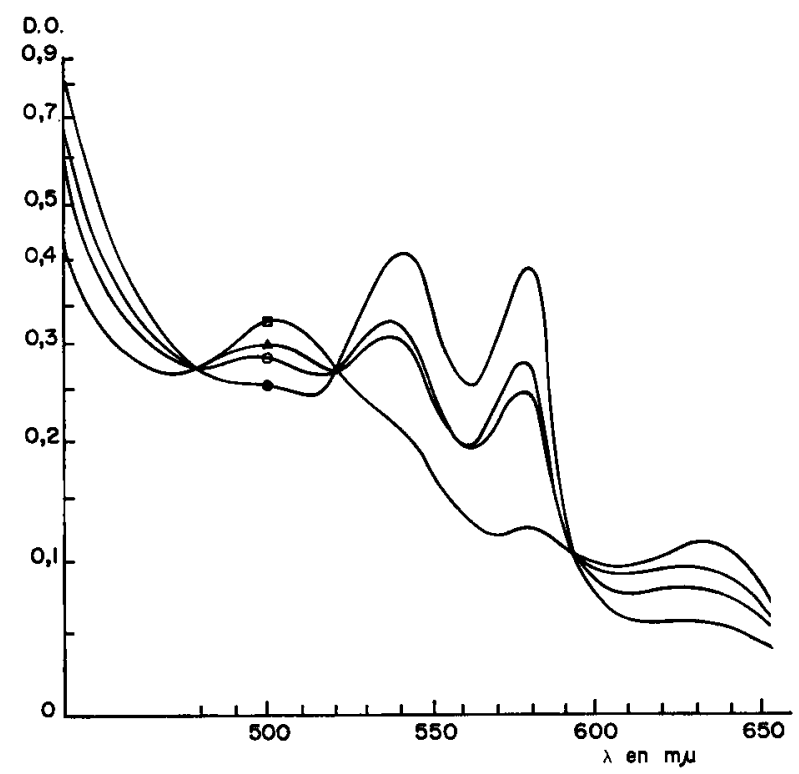

FIG. 4. - Spectres d'absorption de solution de myoglobine après incubation d différentes conditions de température et de $p H$

$\mathrm{pH} 5,537^{\circ}-43^{\circ} 4$ heures

O $\mathrm{pH} 5,537^{\circ}$ I heure $\triangle \mathrm{pH} 6,537^{\circ}-43^{\circ} 4$ heures

- $\mathrm{pH} 6,537^{\circ} \mathrm{I}$ heure 
$630 \mathrm{~m} \mu$, ce qui traduit la formation de metmyoglobine. L'étude spectrale des extraits obtenus après chromatographie sur carboxyméthyl-cellulose montre que la conjonction bas $\mathrm{pH}$ (5,5 minimum) et température élevée $\left(45^{\circ}\right.$ maximum) entraîne une oxydation et non une dénaturation de la myoglobine.

Il convient de remarquer, en effet, que les spectres d'absorption des extraits présentent trois points isobestiques à $474 \mathrm{~m} \mu$, à $525 \mathrm{~m} \mu$, à $597 \mathrm{~m} \mu$ et que les maxima de l'oxymyoglobine à $544 \mathrm{~m} \mu$ s'estompent progressivement, tandis que les maxima de la metmyoglobine à $500 \mathrm{~m} \mu$ et à $630 \mathrm{~m} \mu$ se manifestent très nettement. L'existence de deux points isobestiques et 1'apparition des caractéristiques spectrales de la metmyoglobine montrent que la quantité totale de myoglobine est constante et que le pourcentage de metmyoglobine augmente au fur et à mesure que le $\mathrm{pH}$ diminue et que la température s'élève. L'oxydation de la myoglobine semble particulièrement favorisée par l'élévation de la température (fig. 4). En effet, après une durée d'incubation de 4 heures à $43^{\circ}$ les spectres sont semblables, que le pH soit de 5,5 ou 6,5 . Toutefois, plus le $\mathrm{pH}$ est élevé, plus la vitesse d'oxydation est ralentie.

\section{2. Caractéristiques électrophorétiques.}

La figure 5 représente, après coloration à la benzidine, les électrophorégrammes d'extraits bruts et de solution obtenus par chromatographie sur carboxyméthylcellulose qui, dans les deux cas, ont été ajustés à $\mathrm{pH} 5,5$, puis maintenus à une température de $45^{\circ} \mathrm{C}$ pendant 4 heures. Les électrophorégrammes des solutions obtenues par chromatographie sur CMC se caractérisent par l'absence de la bande correspondant à l'hémoglobine résiduelle, mais le traitement thermique ne provoque manifestement aucune modification du comportement électrophorétique de la myoglobine. Avant et après le traitement, la myoglobine présente trois bandes anodiques, la plus importante ayant la vitesse de migration la plus lente. Il apparaît donc que la modification du degré d'oxydation de la myoglobine n'influence pas la rapiditéde la migration électrophorétique.

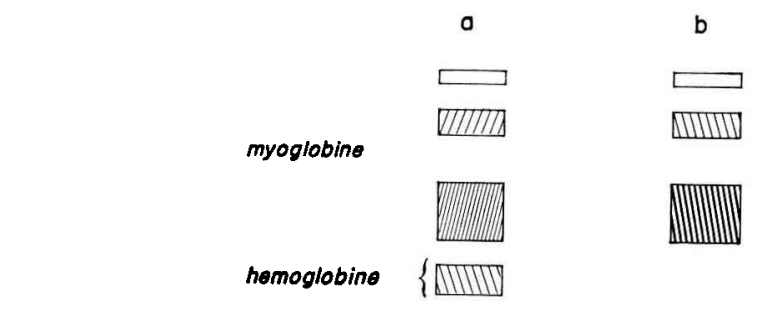

FIG. 5. - a) Électrophorégramme de l'extrait brut (coloration par la benzidine) b) Électrophorégramme de la solution de myoglobine (coloration par la bendizine)

\section{Conséquences technologiques}

Le délai nécessité par l'abattage et la préparation de la carcasse ne peut guère, dans les conditions pratiques, être inférieur à 45 minutes. La température musculaire est alors comprise entre $39^{\circ} \mathrm{C}$ et $43^{\circ} \mathrm{C}$. Lorsque le $\mathrm{pH}$ diminue rapidement 45 minutes après l'abattage sa valeur est inférieure ou égale à 5,5 . Il est donc manifeste qu'une conjonction $\mathrm{pH}$-température néfaste est alors inévitable. Par suite des 
différences de vitesses de refroidissement des muscles en fonction de leur localisation anatomique, la réfrigération rapide est surtout efficace pour les muscles superficiels (fig. 6). D'un point de vue pratique, il semble donc que, dans les cas extrêmes du moins, la réfrigération rapide des carcasses ne permet pas de pallier les défectuosités de rétention d'eau et de coloration. Par contre, dans le cas de muscles superficiels présentant une chute de $\mathrm{pH}$ post mortem relativement lente, la rapidité et l'intensité de la réfrigération peuvent alors exercer un effet bénéfique en abaissant suffisamment la température musculaire.

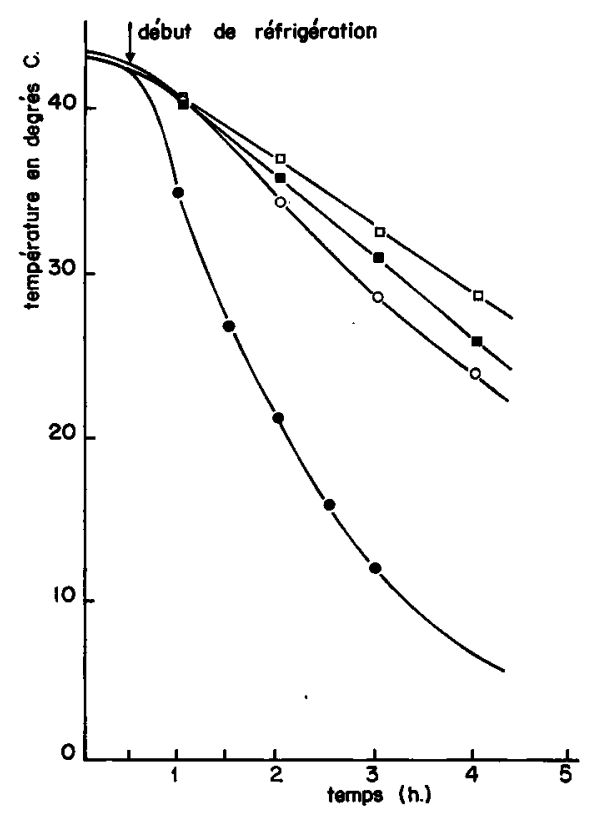

FIG. 6. - Évolution de la température interne des muscles Adductor et Longissimus dorsi pour des températures ambiantes de $+4^{\circ} \mathrm{C}$ et $-20^{\circ} \mathrm{C}$ $\begin{array}{ll}\text { ․ Adductor }+4^{\circ} \mathrm{C} & \text { - Adductor }-20^{\circ} \mathrm{C} \\ \circ \text { Longissimus dorsi }+4^{\circ} \mathrm{C} & \text { - Longissimus dorsi }-20^{\circ} \mathrm{C}\end{array}$

\section{CONCLUSION}

L'étude de la solubilité des protéines sarcoplasmiques en milieu de faible force ionique dans des conditions variables de $\mathrm{pH}$ et de température permet de préciser les influences respectives de ces deux paramètres, ainsi que la nature de la fraction dénaturée. Bien que cette étude mérite d'être complétée par celle de la solubilité des protéines myofibrillaires elle permet néanmoins d'ores et déjà de prévoir les résultats qui sont susceptibles d'être obtenus au moyen des techniques de réfrigération rapide. $A$ priori, de telles techniques laissent espérer une amélioration possible de la rétention d'eau et de la coloration dans le cas de muscles présentant une évolution post mortem du $\mathrm{pH}$ caractéristique d'une viande de qualité moyenne dans des conditions technologiques traditionnelles. En revanche, il ne semble pouvoir en être de même pour les 
muscles dont la glycogénolyse post mortem est anormalement rapide. Seule, la maîtrise de la glycogénolyse permettrait donc, en définitive, d'éviter systématiquement une importante dénaturation des protéines sarcoplasmiques et d'améliorer, par conséquent, la capacité de rétention d'eau et la coloration des viandes de porc.

Reçu pour publication en juillet 1968.

\author{
SUMMARY
}

\title{
EFFECT OF TEMPERATURE AND PH ON PHYSICOCHEMICAL PROPERTIES OF SARCOPLASMIC PROTEINS OF PORK MUSCLE. \\ PRACTICAL CONSEQUENCES
}

This experiment was undertaken in order to investigate the effect of temperature and $\mathrm{pH}$ on the solubility of sarcoplasmic proteins and on the spectral and electrophoretic properties of myoglobin in the Longissimus dorsi muscle of pig.

Sarcoplasmic proteins were incubated for 4 hours at various temperatures in citrate + phosphate or phosphate-buffer $0.2 \mathrm{M}$ solutions, so as to obtain $\mathrm{pH}$ values ranging from 5.5 to 6.7 . The soluble proteins were characterized by FoLIN-LowRY's method. Starch-gel electrophoresis was performed according to Poulik's technique.

The denaturation of sarcoplasmic proteins is the greatest for $\mathrm{pH}$ values from 5.5 to 5.8 and temperature higher than $40^{\circ} \mathrm{C}$. Starch-gel electrophoresis shows that the denaturated proteins are mainly creatine kinase and proteins with fast cathodic migration.

The analyses of myoglobin absorption spectra shows that the combination of low $\mathrm{pH}$ value and high temperature induces oxydation and no denaturation of myoglobin. From a practical viewpoint, our results indicate that for muscles with rapid post mortem $\mathrm{pH}$ fast cooling of carcasses would not allow to make up for the deficiency in water retention and coloration resulting from the denaturation of muscular proteins. On the contrary, fast and severe cooling can be beneficial for muscles with muscles with slow $\mathrm{pH}$ fall.

\section{RÉFÉRENCES BIBLIOGRAPHIQUES}

Awad E. S., Kotite L., 1966. Camel myoglobin. Biochem. J., 98, 909-914.

Bendall J. R., Wismer-Pedersen J., I962. Some properties of the fibrillar proteins of normal and watery pork muscle. J. Food Sci., 27, 144-159.

Bendali J. R., Hallund O., Wismer-Pedersen J., 1963. Post morten changes in the muscles of Landrace pigs. J. Food Sci., 28, I 56-162.

Briskey E. J., Wismer-Pedersen J., I $96 \mathrm{I}$. Biochemistry of pork muscle structure. I. Rate of anaerobic glycolysis and temperature change versus the apparent structure of muscle tissue. $J$. Food Sci., 26, 297305 .

Charpentier J., Goutefongea R., I 963 . Comportement électrophorétique des protéines sarcoplasmatiques du muscle de porc normal et exsudatif. Ann. Biol. anim. Bioch. Biophys., 8, 381-389.

Goldspink G., McLoughlin J. V., r 964 . The effect of temperature on the solubility of the sarcoplasmic proteins in relation to colour changes in post rigor muscle. Irish. J. Agric. Res., 8, 9-16.

GoutefongeA R., 1967. Contribution à l'étude du pouvoir de rétention d'eau de différentes fractions musculaires chez le porc normal et exsudatif. XIII' Congrès des Inst. de Recherches sur la Viande. Rotterdam, 1967 .

Kastenschmidt L. L., Briskey E. J., Hokkstra W. G., ig64. Prévention of pale, soft, exsudative porcine muscle through regulation of ante mortem environmental temperature. $J$. Food Sci., 29, $210-217$.

KRZYWICKI K., WISMER-Pedersen J., 1962. Some properties of sarcoplasmic proteins of normal and watery pork muscle. 8th Meeting Meat Rrs. Instit. Moscou.

Lowry O. H., Rosebrough N. J., FARR A. L., Randall R. J., r95r. Protein measurement with the Folin Phenol reagent. J. Biol. Chem., 198, 265-275.

McLoughlin J. V., I963. The effect of rapid post mortem $\mathrm{pH}$ fall on the extraction of the sarcoplasmic and myofibrillar proteins of post rigor muscle. Iroish J. Agric. Res., 2, 11 5-I 24. 
McLoughlin J. V., Goldspink G., 1963. Post morlem changes in the colour of pig Longissimus dorsi muscle. Nature, 198, $584-5^{85}$.

Morettr J., Boussier G., JAYLe M. F., I957. Réalisation technique et premières applications de l'électrophorèse sur gel d'amidon. Bull. Soc. Chim. Biol., 88, 593-605.

Poulik M. D., I957. Starch-gel electrophoresis in a discontinuous system of buffers. Nature, 180, $1477^{-}$ I479.

SAYRE R. N., BRISkeY E. J., Ig63. Protein solubility as influenced by physiological conditions in the muscle. J. Food Sci., 28, 675-679.

Scopes R.K., I964. Solubilities of muscle proteins. Biochem. $J$, 91, 20I-207. 